\title{
Prevalance of dentigerous cyst reviewed from radiographic examination at Dental Hospital Hasanuddin University
}

\author{
Barunawaty Yunus ${ }^{*}$, Winda Brisbania
}

Abstract

Objective: This research is aimed to find out the prevalence of dentigerous cyst by Radiographic Examination at Dental Hospital Hasanuddin University.

Material and Methods: The type of this research is descriptive observational with cross sectional study design. The population study was all periapical radiographic photo data from all the patients at Dental Hospital Hasanuddin University from septermber 2017September 2018. The data obtained was processed in the form of tables and diagrams.
Results: The results showed that the prevalence of dentigerous cyst based on the cyst type is mostly found in the sentral type with a percentage of $77.8 \%$. The highest percentage based on the gender was found in male with a percentage of $66.7 \%$. Based on age range the highest percentage is $26-45$ years old with a percentage of $66.7 \%$ and based on the the jaw location, the results showed more than half of the dentigerous cyst occurred in the mandible with a percentage of $88.9 \%$. Conclusion: Dentigerous cyst was mostly found in males with a percentage of $66.7 \%$. Based on age groups, dentigerous cyst was mostly found in adults with the age range of 26-45 years.
Department of Dental Radiology, Faculty of Dentistry, Hasanuddin University, Makassar, Indonesia
*Correspondence to: Barunawaty Yunus, Faculty of Dentistry, Hasanuddin University, Makassar, Indonesia

barunawaty@yahoo.com

Received: 5 August 2017

Revised: 26 February 2019

Accepted: 28 February 2019

Available Online 1 August 2019

Keywords: Dentigerous cyst, Radiography

Cite This Article: Yunus B, Brisbania W. 2019. Prevalance of dentigerous cyst reviewed from radiographic examination at Dental Hospital Hasanuddin University. Journal of Dentomaxillofacial Science 4(2): 87-91. D0I: 10.15562/jdmfs.v4i2.861

\section{Introduction}

Cyst is a pathological cavity that contains liquid or semiliquid, not caused by the accumulation of pus. Cysts can be lined by the epithelium, which may cause the enlargement of intraoral and extraoral that can clinically resemble a benign tumor. ${ }^{1}$

Cysts that originated from the rest of the toothforming tissue called crista odontogenic, can also be considered a cyst that its formation has no relation with the tooth-forming tissue called nonodontogenic. Cysts that are located in the jaw bone may have an epithelium that originated from the odontogenic epithelium, such as from a residual dentin or email organ. ${ }^{2}$

Odontogenic cyst are pathological cavities that consists of fluid, coated by the epithelium and collagen tissue, which originates from the odontogenic epithelium. Epithelium in the formation of cysts in the jaw comes from the remaining tissue of the dental lamina and residual cells from serres (cell rests of serres) and contraction of the email epithelium that arranges the dentin follicles due to the effect of dentition and residual cells from malassez (cell rest of malassez). Meanwhile, the non-odontogenic cyst is the mucous cyst that its formation has no relation with the tooth-forming tissue. Non-odontogenic cysts originate from the ectoderm that is involved in the facial tissue formation. ${ }^{2,3}$

Dentigerous cyst is always connected with the crown of the teeth that has not erupted yet or a growing tooth. Dentigerous cysts is also known as the follicular cysts because it comes from the email organ or teeth follicle. Dentigerous cyst surrounds the crown of the teeth that has not erupted yet and is attached to the teeth along the cervical region. Dentigerous cysts is usually formed in impacted tooth and permanent supernumerary tooth., ${ }^{2,45}$

The etiology for dentigerous cyst is usually related with an impacted tooth, tooth with a delayed eruption, tooth development, and odontoma. ${ }^{6}$

There are two theories found in the formation of cysts found. The first theory states that cyst is caused by the accumulation of liquid between the induced email epithelium and crown of the teeth. The pressure of the liquid pushes the reduced email epithelium proliferation into the cyst that is attached to the cement-enamel junction and crown of the teeth. $^{3}$

The second theory states that a cyst begins with 
the damage of the stellate reticulum, eventually forming liquid between the inside part of the email epithelium and the outside part of the email epithelium. The pressure of that liquid will push the outside of the epithelium proliferation email that leaves behind an attachment on the teeth, specifically on the cement-enamel junction, which then causes the inside of the email epithelium to be pushed into the upper surface of crown. ${ }^{3}$

The cyst is formed surrounding the crown and attached on the cement-enamel junction of the teeth. When the formation has been completed perfectly, the crown will protrude into the lumen, and the roots will elongate to the outside part of the cyst. ${ }^{3}$

In every theory, liquid causes the proliferation of the cyst because of the substance inside the hyperosmolar that is resulted from a cellular breakdown and other cell products, eventually causing the osmotic gradient to pump the liquid into the lumen of the cyst. ${ }^{3}$

Dentigerous cyst itself is classified based on the location on the crown of the teeth to 3 types, which are the central type, lateral type and circumferential types. The central type is the type that is most commonly found. For this type, the cyst surrounds the crown symmetrically, moving the teeth to the opposite direction of the normal eruption direction. The lateral type cyst usually grows laterally (on the mesial and distal sides) along the surface of the tooth's root, extends far from teeth, and some of the cyst surrounds the crown of the teeth, which causes the teeth to tilt to the direction that is not surrounded by the cyst. For the circumferential type, the cyst surrounds the crown of teeth and extends along the tooth's root, eventually making the tooth's root located inside the cyst. ${ }^{5}$

The clinical description of a dentigerous cyst involves a tooth usually impacted or erupted late. Most of them is related with the third molar mandibular, as well as the maxillary canines, third molar maxillary, and 2 premolar mandibulars. $8 \otimes$ Nevertheless, this cyst can still occur in all unerupted teeth, where the crown of the teeth has a cyst lumen. ${ }^{2}$

A dentigerous cyst that has not experienced complications yet likewise others cysts will not trigger symptoms until the enlargement becomes clearer. Although symptoms are usually absent, late teeth eruption may increase the indication of a dentigerous cyst occuring. ${ }^{2}$

Clinically, the lesion appears as a swelling of the alveolar linger (ridge) above the location of the erupting tooth. When the circumcoronal cyst cavity contains blood, the swelling appears purple or really blue, eventually making it called hematoma eruption. Dentigerous cysts are usually solitary, if multiple may occur together with the nevoid basal cell carcinoma syndrome. ${ }^{2}$

Cysts may have a variety of sizes, from one that takes a slow time to enlarge on the pericoronal pouch until the one that covers the entire body and ramus mandible as well as some of the jaw bone. Cysts on the lower jaw usually is usually found to be bigger than on the upper jaw. ${ }^{2}$

The small dentigerous cyst rarely causes clinical symptoms, but big cysts will expand to the bone and cause intra-oral swelling, outer-oral swelling, or both. Dentigerous cyst are usually asymptomatic unless they are very big $(10-15 \mathrm{~cm})$ or if a secondary infection takes place, eventually causing pain. Secondary infections usually happen. It may also cause jaw expansion. ${ }^{6}$

This dentigerous cyst can also cause the face to be not symmetrical, or secondary infections, which may cause pain.

The normal size of the follicular space is less than $2,5 \mathrm{~mm}$ based on intraoral radiograph and 3 $\mathrm{mm}$ based on panoramic radiograph with the bigger space being considered as the cyst.

Dentigerous cyst can be easily identified radiologically because the radiographic picture has a special characteristic. Radiographically, dentigerous cyst shows a radiolucent area. Radiolucency is usually seen to be clearly bounded and firm, unilocular or multilocular, pericoronal radiolucency, and with a sclerotic border around the crown of the teeth that has not erupted. Radiolucent area starts from the cervical border. The infected cyst shows an unclear boundary. A big dentigerous cyst gives an impression of a multilocular process because of the persistence of the trabecular bone that is seen to be radiolucent. ${ }^{2,5}$

Radiographically, the cyst's internal aspect looks radiolucent except for the crown of the teeth involved. The cyst appears translucent and compressible when the cyst's expansion causes cortical bone resorption.

Movement or displacement of a tooth that has not erupted with all sorts of positions often occurs and can be found in the maxilla or mandible. On the mandibular area, the radiolucent picture involved can enlarge into the superior from the third molar region into the ramus or through anteri or-inferior along the mandibular body. On the upper jaw bone dentigerous cyst involving the can ine region, expansion into the maxillary sinus or towards the orbital wall can be found as well as the extension into the nasal fossa. The third maxilla molar can extend distally and superiorly, usually related with the space of the maxillary sinus. ${ }^{2}$ The histopathologic picture of a dentigerous cyst generally consists of a thin layer of connective tissue wall, coated with a non-keratinized flat squamous 
epithelium that unites with the rest of the email epithelium, covering or adhering to the neck of the tooth's crown.

On the uninflamed dentigerous cyst, the epithelial layer consist of 2-4 layers of un-keratinized cuboidal epithelial cells and the space between the tissue and epithelium is flat. The walls of fibrous tissue are loosely arranged and consists of quite a big amount of glycosaminoglycan's base substance. ${ }^{3-5}$

On inflamed cysts or cysts that experience secondary infections, the epithelial layers become hyperplasia, the development of rete peg from the squamous epithelium will occur. On inflamed dentigerous cysts, the fibrous walls are denser of collagen, eventually making it more elastic, with various kinds of infiltration from chronic inflammatory cells. The surface that undergoes keratinization is often seen. ${ }^{3,5}$ In some cases, dentigerous cyst is found to contain melanin pigment and melanocyte on the layer wall of the epithelium. Sometimes, the wall of epithelium appears with or without a smooth keratinization surface and a lot of granular from the melanin pigment distributed in the basal cell at the epithelium layer. Mucus cells can spread in the epithelial of the dentigerous cyst. Inside the fibrous wall of a cyst, a columnar cell or cuboidal cel that has cilia and a collection of small sebaceous cells. Elements of the mucus, cilia, and sebaceous may hint to the potential of an odontogenic epithelial layer in a dentigerous cyst. ${ }^{3,4}$

Dental radiography is used to assist diagnosis and treatment plans for various abnormalities or diseases of the oral cavity, one of which is cyst. In the world of dentistry, radiographic examination has an important role in the diagnosis process. Almost all the treatments in the dentistry world require radiographic support data to achieve the optimal results.

Radiography in dentistry is divided into two, which are intraoral radiography and extraoral radiography. Extraoral radiography and intraoral radiography as well as its types have a specific usability and function. The resulting picture of intraoral or extraoral radiography is very important for dentists, especially to see.

One of the dental radiographs is periapical radiography. This radiography is a type of intraoral radiography that describes three until four teeth with surrounding. Periapical radiography is divided into two techniques, parallel and bisecting. During the parallel technique, the film is placed on the film holder and positioned parallel to the axis of the tooth, whereas during the bisecting technique, parallel and bisecting. During the parallel technique, the film is placed on the film holder and positioned parallel to the axis of the tooth, whereas during the bisecting technique, the film is placed as close as possible to the palatal/lingual surface of the tooth.

\section{Material and Methods}

The type of this research is descriptive observation with cross sectional study design. The population study was all the periapical radiographic photo data collected from all the patients at Dental Hospital Hasanuddin University in the span of one year, from September 2017-September 2018.

This research used periapical radiographic photo data that consists of male and female subjects, as well as consisting of all age groups. The data that can be obtained through periapical photo are the types and locations of the dentigerous cyst itself.

Obtaining data on this research was done by seeing the periapical radiographic photo at Dental Hospital Hasanuddin University in the Department of Radiology with no further clinical examinations done. All the data were collected, written, and converted into a table and diagram, in addition to getting analyzed.

\section{Results}

Based on the observational data, there were 2.563 periapical radiographic photo data at Dental Hospital Hasanuddin University in the department of radiology in the span of one year, from September 2017-September 2018, and the samples obtained totals up to 9 samples.

Table 1 and figure 1 shows the distribution of dentigerous cyst based on gender. Based on the data found, there are more males compared to females, with as much as $66.7 \%$ in males and $33.3 \%$ in females.

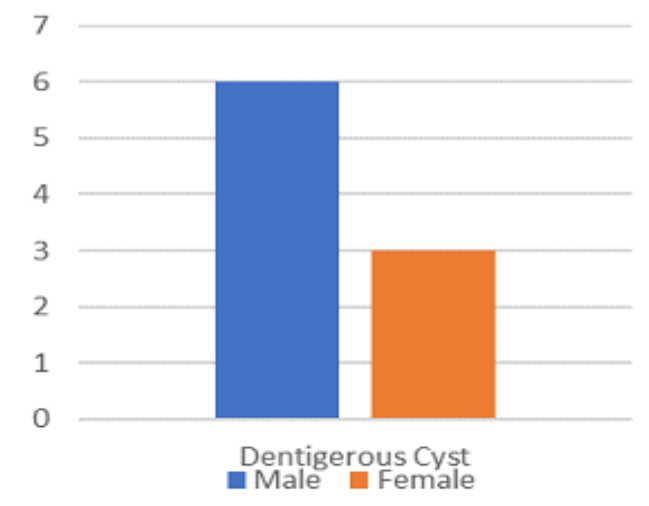

Figure 1 Dentigerous cyst based on gender 
Table 1 Distribution of dentigerous cyst based on gender

\begin{tabular}{lcc}
\hline Gender & Frequency & Percentage \\
\hline Male & 6 & 66.7 \\
Female & 3 & 33.3 \\
Total & 9 & 100 \\
\hline
\end{tabular}

Table 2 Distribution of dentigerous cyst based on age groups

\begin{tabular}{lcc}
\hline Age groups & Frequency & Percentage \\
\hline Children & 0 & 0 \\
Teenagers (12-25 years) & 1 & 11.1 \\
Adults (26-45 years) & 6 & 66.7 \\
Elders (>45 years) & 2 & 22.2 \\
Total & 9 & 100 \\
\hline
\end{tabular}

Table 3 Distribution of dentigerous cyst based on jaw location

\begin{tabular}{lcc}
\hline Age groups & Frequency & Percentage \\
\hline Maxilla & 1 & 11.1 \\
Mandible & 8 & 88.9 \\
Total & 9 & 100 \\
\hline
\end{tabular}

Table 4 Distribution of dentigerous cyst based on age groups

\begin{tabular}{lcc}
\hline Cyst type & Frequency & Percentage \\
\hline Central & 7 & 77.8 \\
Lateral & 1 & 11.1 \\
Circumferential & 1 & 11.1 \\
Total & 9 & 100 \\
\hline
\end{tabular}

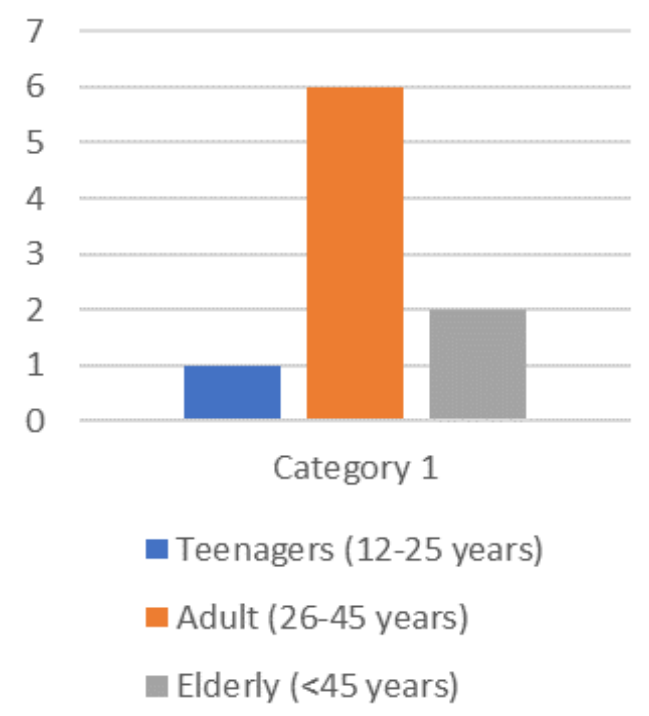

Figure 2 Dentigerous cyst based on age groups

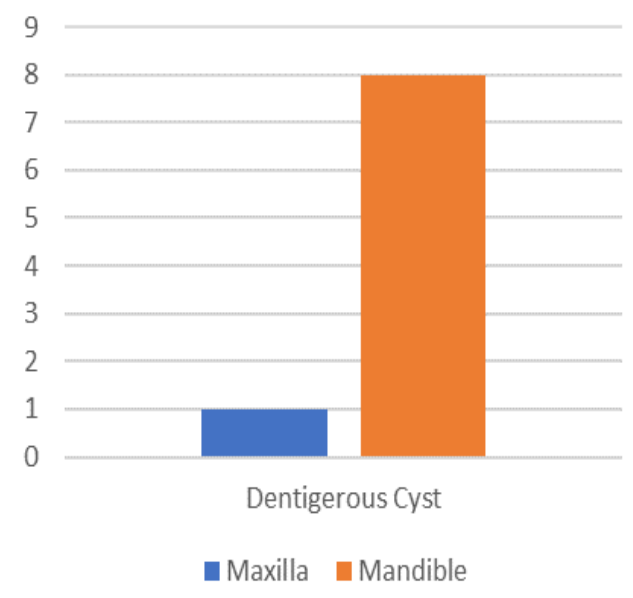

Figure 3 Dentigerous cyst based on jaw location

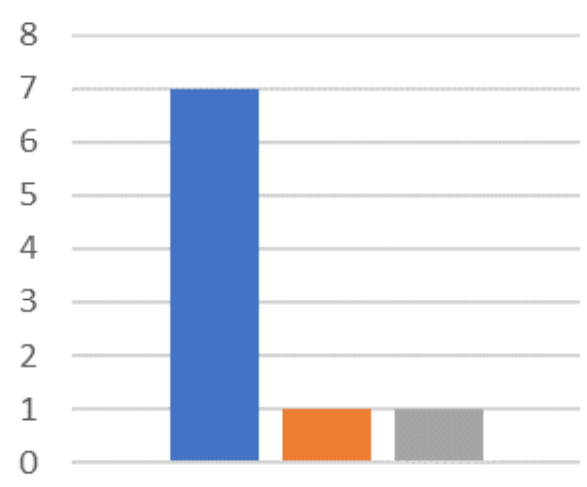

Kista Dentigerous

Tipe Sentral

Figure 4 Dentigerous cyst based on cyst type

Table 2 and figure 2 from the total samples taken, dentigerous cyst is not found in children, but it is found in teenagers with a percentage of $11.1 \%, 66.7 \%$ in adults, and $22.2 \%$ in elders. This proves that dentigerous cyst is mostly found in adults, with an age range of $26-45$ years old.

On table 4 and figure 4, the distribution of the sample is shown based on the type of the cyst. From the result of this research, the central type is the type mostly found with a percentage of $77.8 \%$, while the lateral type is found with as much as $11.1 \%$ and the circumferential type with as much as $11.1 \%$.

\section{Discussion}

Based on this research, dentigerous cyst is mostly found in males compared to females with a percentage of $66.7 \%$. This research is in line with the research by Selvamani. ${ }^{7}$ in India and Acikgoz. ${ }^{8}$ in Turkey, with the male's percentage being higher 
than the female's percentage.

In Shear's opinion, it might be a temptation to assume that the less frequent occurrence in women is due to them having a lower prevalence of unerupted teeth. In surveys of large series of radiographs, both groups found that there was no gender difference in the frequency of unerupted teeth. However, there was a difference in the frequency of impactions between black men and women, although there was no significant difference between men and women in whites or in the total sample. These data suggested that there was another factor that has not been identified, but possibly innate, that may influence the development.

Some studies from a few countries have reported that dentigerous cyst may happen to every age group, but is mostly found in the third and fourth decades of life. However, it is not rarely found in the first, second, fifth, and sixth decades of life either. ${ }^{8}$

Acknowledging the fact that dentigerous cyst is an odontogenic cyst, Selvamani et al. ${ }^{7}$ research in India states that a person who is either a teenager or an adult is more prone to obtaining an odontogenic cyst compared to other age groups.

From the result of the research gathered, most dentigerous cyst is found in the mandible with a percentage of $88.9 \%$. Based on theory, the frequency of dentigerous cysts at these sites can be explained by the fact that the lower third molars and upper canines are the most commonly impacted teeth. Therefore, the location of the dentigerous cyst will follow.?

Dentigerous cyst is divided into three types, the central type, lateral type, and circumferential type. The most common type of dentigerous cyst is the central type, where the cyst surrounds the crown symmetrically. According to this research, the appropriate results were obtained, with the central type being found as much as $77.8 \%$.

\section{Conclusion}

Nine cases of dentigerous cyst were found at Dental Hospital Hasanuddin University from September 2017-September 2018. Dentigerous cyst was mostly found in males with a percentage of $66.7 \%$. Based on age groups, dentigerous cyst was mostly found on age groups, dentigerous cyst was mostly found in adults with the age range of $26-45$ years. For the jaw location, most of the dentigerous cyst was found in the mandible with a percentage of $88.9 \%$. The most common type of dentigerous cyst was the central type with a percentage of $77.8 \%$.

\section{Acknowledgment}

Thank you to the Dental Hospital Hasanuddin University and Faculty of Dentistry Hasanuddin University that has been very supportive in this research.

\section{Conflict of Interest}

The authors report no conflict of interest.

\section{References}

1. Neville, Damm DD, Allen CM, et al. Oral and maxillofacial pathology 2nd ed. St. Louis: Saunders; 2002. p. 611-619.

2. Oliveira GJ, Jose N, Lois N, et al. Conservative management of a dentigerous cyst secondary to primary tooth trauma. Dent Traumatol 2008;24: 676-679.

3. Mihailova, Nikolov, Slaukov. Diagnostic imaging of dentigerous cysts of the mandible. J IMAB 2008: 1-3.

4. Oliveira GJ, Jose N, Lois N, et al. Conservative management of a dentigerous cyst secondary to primary tooth trauma. Dent Traumatol 2008;24: 676-679.

5. Kambalimath DH. Prevalence and distribution of odonto genic cyst in Indian population: a 10 year retrospective Study. J Maxillofac Oral Surg 2014;13: 10-15.

6. Souza LB. Odontogenic cyst: demographic profile in a Brazilian ppopulation over 38-year period. Med Oral Patol Cir Bucal 2010;15: e583-e590.

7. Selvamani M, Donoghue M, Basandi PS. Analysis of 153 cases of odontogenic cyst in a south indian sample popula tion: a retrospective study over a decade. Braz Oral Res 2012;3.

8. Acikgoz A. Prevalance and distribution of odontogenic and non odontogenic cyst in a Turkish population. Med Oral Patol Cir Bucal 2012;17: e108-e115. 